\title{
OATP1B2 deficiency protects against paclitaxel-induced neurotoxicity
}

\author{
Alix F. Leblanc, ${ }^{1}$ Jason A. Sprowl, ${ }^{2}$ Paola Alberti, ${ }^{3}$ Alessia Chiorazzi, ${ }^{3}$ W. David Arnold, ${ }^{4}$ Alice A. Gibson, ${ }^{1}$ Kristen W. Hong, ${ }^{1}$ \\ Marissa S. Pioso, ${ }^{1}$ Mingqing Chen, ${ }^{1}$ Kevin M. Huang, ${ }^{1}$ Vamsi Chodisetty, ${ }^{1}$ Olivia Costa, ${ }^{2}$ Tatiana Florea, ${ }^{2}$ Peter de Bruijn, ${ }^{5}$ \\ Ron H. Mathijssen, ${ }^{5}$ Raquel E. Reinbolt, ${ }^{6}$ Maryam B. Lustberg, ${ }^{6}$ Lara E. Sucheston-Campbell, ${ }^{7}$ Guido Cavaletti, ${ }^{3}$ \\ Alex Sparreboom, ${ }^{1}$ and Shuiying $\mathrm{Hu}^{1}$ \\ 'Division of Pharmaceutics and Pharmaceutical Chemistry, College of Pharmacy and Comprehensive Cancer Center, The Ohio State University, Columbus, Ohio, USA. ²Department of Pharmaceutical, \\ Social and Administrative Sciences, School of Pharmacy, D’Youville College, Buffalo, New York, USA. ${ }^{3}$ Experimental Neurology Unit and Milan Center for Neuroscience, School of Medicine and Surgery,

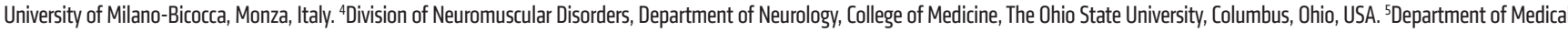 \\ Oncology, Erasmus MC Cancer Institute, Rotterdam, Netherlands. ${ }^{6}$ Department of Internal Medicine, College of Medicine, and ${ }^{7}$ Division of Pharmacy Practice and Science, College of Pharmacy, \\ The Ohio State University, Columbus, Ohio, USA.
}

Paclitaxel is among the most widely used anticancer drugs and is known to cause a dose-limiting peripheral neurotoxicity, the initiating mechanisms of which remain unknown. Here, we identified the murine solute carrier organic aniontransporting polypeptide B2 (OATP1B2) as a mediator of paclitaxel-induced neurotoxicity. Additionally, using established tests to assess acute and chronic paclitaxel-induced neurotoxicity, we found that genetic or pharmacologic knockout of OATP1B2 protected mice from mechanically induced allodynia, thermal hyperalgesia, and changes in digital maximal action potential amplitudes. The function of this transport system was inhibited by the tyrosine kinase inhibitor nilotinib through a noncompetitive mechanism, without compromising the anticancer properties of paclitaxel. Collectively, our findings reveal a pathway that explains the fundamental basis of paclitaxel-induced neurotoxicity, with potential implications for its therapeutic management.

\section{Introduction}

The tubulin poison paclitaxel is among the most widely used drugs in the treatment of a variety of solid tumors, including early-stage and metastatic breast cancer (MBC). Paclitaxel induces a chronic, dose-dependent sensory peripheral neuropathy that is characterized by tingling, numbness, increased sensitivity to cold and touch, and burning pain of the distal extremities, and this side effect occurs with an incidence of up to $70 \%-80 \%$ in patients with $\mathrm{MBC}$ (1). With cumulative dosing, the painful symptoms increase in severity and can persist for years (2), or even cause a lifelong functional impairment that impacts quality of life (3). This toxicity may also lead to therapy cessation, which may impact clinical outcomes.

The mechanistic basis of this side effect remains uncertain (4), although prior studies have demonstrated that paclitaxel induces injury to sensory neurons, morphological and biochemical alterations in dorsal root ganglia (DRG) satellite cells, hyperplasia/hypertrophy of macrophages in the peripheral nervous system, and increases microglial and astrocyte activation within the spinal cord $(5,6)$. Paclitaxel also causes an acute pain syndrome that develops within 1-3 days of paclitaxel administration, and that resolves within 1 week. This acute syndrome is possibly caused by activation of Toll-like receptor 4 (TLR4) in the spinal dorsal horn

Conflict of interest: The authors have declared that no conflict of interest exists. Submitted: July 7, 2017; Accepted: November 28, 2017.

Reference information: / Clin Invest. 2018;128(2):816-825.

https://doi.org/10.1172/JCI96160. and DRG (7), the main site of paclitaxel uptake within the nervous system (8). Interestingly, prospective clinical studies have demonstrated that patients experiencing the acute pain syndrome following the first administration of paclitaxel are those who subsequently develop chronic neuropathy (9).

Over the last 3 decades, various approaches have been proposed to predict, prevent, and/or treat paclitaxel-induced peripheral neuropathy (10). The predictive strategies have predominantly focused on the search for hereditary biomarkers that could identify patients at increased risk of toxicity through candidate gene or genome-wide association studies $(5,11,12)$. However, the findings from these studies done to date have identified nonoverlapping single or pathway biomarker associations that preclude immediate clinical implementation. In addition, the decision to act on a toxicity biomarker is hampered in many diseases by the lack of available alternative treatments to replace paclitaxel and/ or the need for a patient-tailored reduction in the paclitaxel dose to prevent toxicity, which will have negative effects on disease management. Against this background, the development of preventative strategies that could effectively afford tissue protection during paclitaxel treatment is urgently needed. Although preventative interventions have been proposed, many of these have not been evaluated in animal models or humans. Moreover, to date there have been more than 40 randomized controlled clinical trials of agents to prevent or treat peripheral neuropathy, and these trials have generally failed to provide convincing evidence for a clinically beneficial agent (13). Here, we report that paclitaxel accu- 
A

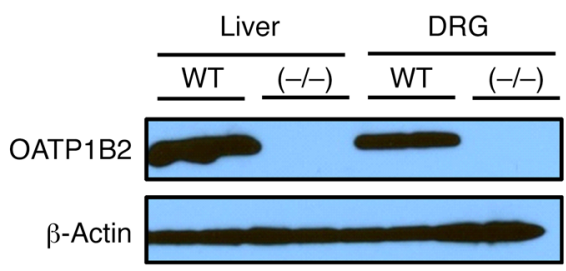

C

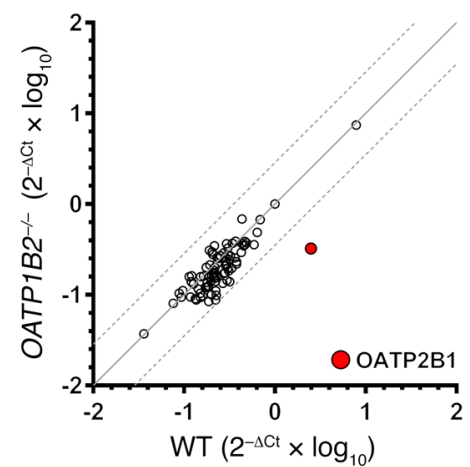

B
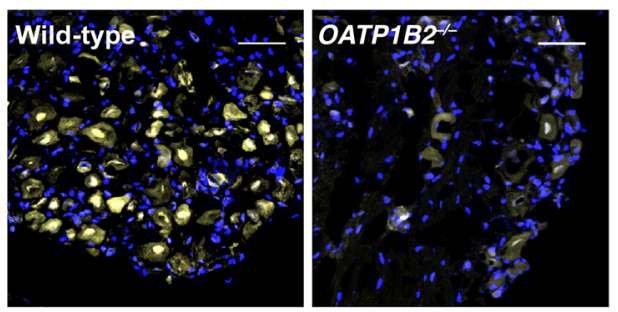

D

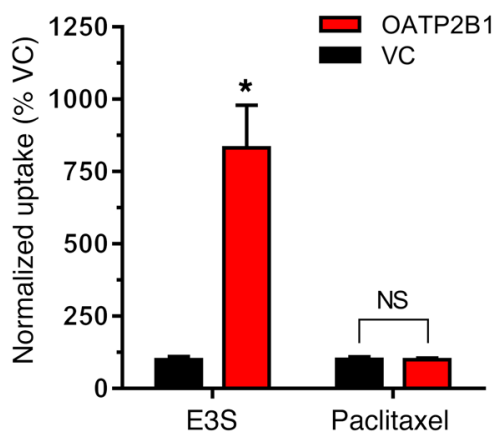

Figure 1. Phenotypic characterization of OATP1B2--- mice. (A) Comparative expression of OATP1B2 protein in liver and DRG at baseline of wild-type (WT) and OATP1B2-- (-/-). (B) Detection of OATP1B2 by immunofluorescence in DRG of wild-type and OATP1B2-- mice. OATP1B2 is depicted by yellow fluorescence, whereas DNA is depicted in blue (DAPI). Scale bars: $50 \mu \mathrm{m}$. (C) Comparative expression of 84 transporter genes in DRG at baseline of wild-type and OATP1B2 ${ }^{-1-}$ mice ( $n=3$ samples per group). Each symbol represents an average reading for a single gene, the solid line is the line of identity, and the dotted lines represent the $95 \%$ confidence intervals. The colored symbol represents the transporter gene for OATP2B1 (SLCO2B1). (D) Lack of paclitaxel transport by OATP2B1 in HEK293 cells transfected with an empty vector (VC) or OATP2B1 ( $2.5 \mu \mathrm{M}$; 5 -minute incubations). Overexpression of the transporter was confirmed by evaluating the uptake of estrone-3-sulfate (E2S). Data represent the mean of triplicate observations from experiments performed on 2 separate occasions, and are expressed as average percentage of uptake values in cells transfected with an empty vector (VC) with error bars representing SD. *Denotes significant differences from VC $(P<0.05)$, and NS denotes not significant, as evaluated with an unpaired 2-sided Student's $t$ test with Welch's correction.

mulates in cells through a process that is mediated by OATP1B2, that paclitaxel induces neurotoxicity in mice in an OATP1B2dependent manner, and that these phenotypes can be reversed by a pretreatment with the tyrosine kinase inhibitor (TKI), nilotinib.

\section{Results}

OATP1B2 as a putative paclitaxel transporter in mouse neurons. Paclitaxel is known to extensively accumulate in DRG and can be taken up into cells via a process mediated by specific organic anion-transporting polypeptides (OATPs), in particular OATP1B1 (SLCO1B1) and OATP1B3 (SLCO1B3) in humans (14-17) and OATP1B2 (Slco1b2) in rodents (18). To directly demonstrate a contribution of this transport system to paclitaxel toxicity, we used a mouse model with and without deletions of OATP1B2 (OATP1B2 $2^{-/-}$mice). Analysis of livers and DRG from wild-type mice revealed that OATP1B2 is highly expressed in both tissues as based on immunoblotting (Figure 1A) and immunofluorescence in DRG (Figure 1B). The expression of 84 ATP-binding cassette $(\mathrm{ABC})$ transporter and solute carrier (SLC) genes (Supplemental Table 1; supplemental material available online with this article; https://doi.org/10.1172/JCI96160DS1) was not substantially changed in DRG from OATP1B2 $2^{-/}$mice compared with tissues obtained from wild-type animals, with the exception of a downregulation of transcripts of the related transporter OATP2B1 (Figure 1C). The functional impact of this observation is likely limited, considering that paclitaxel was not found to be a transporter substrate of OATP2B1 (Figure 1D).
Role of OATP1B2 in paclitaxel-induced neuropathy. To determine the role of OATP1B2 in paclitaxel-induced neuropathy in vivo, we first performed comparative pharmacokinetic studies of paclitaxel in wild-type mice and OATP1B2 $2^{-/}$mice (19). These studies demonstrated that the average systemic exposure, measured by the area under the curve (AUC) in plasma, after the administration of a clinically relevant dose of paclitaxel was only modestly increased in $O A T P 1 B 2^{--}$mice (Figure 2A), whereas the observed terminal halflives of paclitaxel were not dependent on mouse genotype (Supplemental Table 2). This suggests that, from a pharmacokinetic perspective, these mice are an appropriate model of human patients (18), with the knowledge that potentially genotype-dependent differences in systemic exposure are unlikely to directly affect the extent to which paclitaxel can induce peripheral neuropathy. We also found that, as reported previously with docetaxel (15), genetic deficiency of OATP1B2 in mice results in decreased liver uptake of paclitaxel, as well as decreased uptake into DRG (Figure 2B).

This mouse model was subsequently used to first determine the role of OATP1B2 in paclitaxel-induced neuropathy on the basis of a test that evaluates mechanical allodynia, a widely used measure of acute paclitaxel-induced neuropathy in mice $(2,7,20)$. Our results using this test suggest that wild-type and $O A T P 1 B 2^{---}$mice do not show intrinsic differences in sensitivity to mechanical stimuli at baseline (Figure 2C). However, wild-type mice experienced significantly increased sensitivity to mechanical stimulation after administration of paclitaxel, where the force to induce a response decreased by more than $50 \%$ within 2 days after administration of 
A

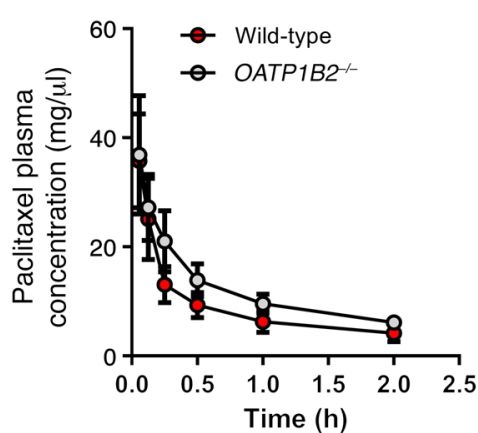

B

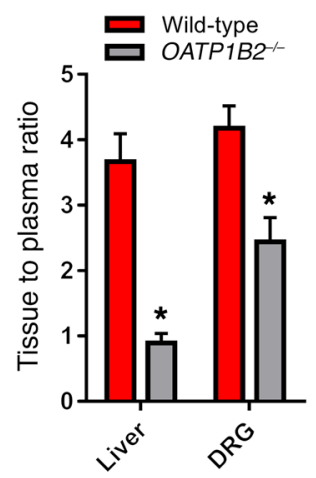

C

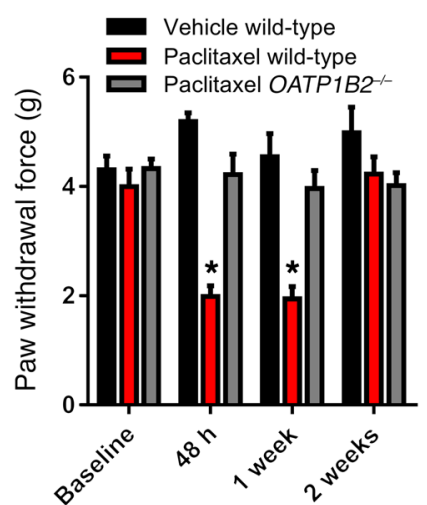

D

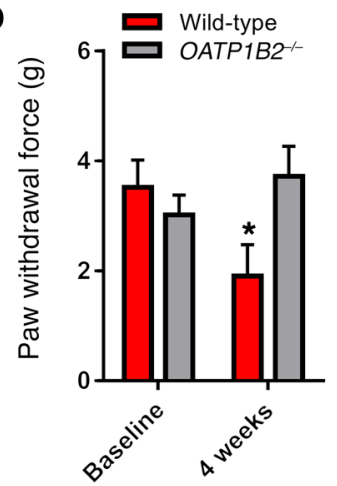

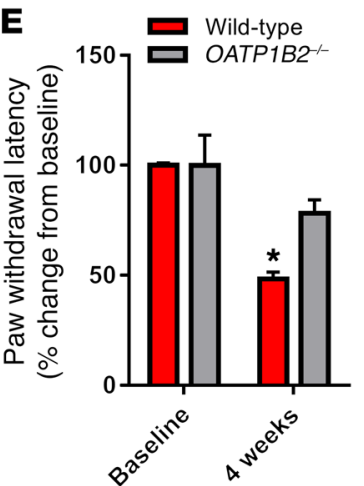

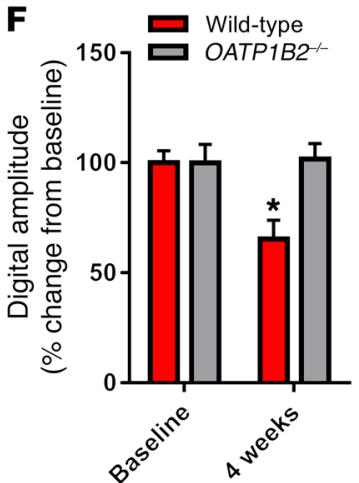

Figure 2. Influence of OATP1B2 loss on paclitaxel disposition and neurotoxicity. (A) Plasma concentration-time curves and (B) tissue distribution ratios of paclitaxel after an i.v. dose of $10 \mathrm{mg} / \mathrm{kg}$. Mechanical allodynia after 1 or 2 doses of paclitaxel (10 mg/kg, i.v.; time 0 and 72 hours) (C), or 4 doses (70 mg/ kg, i.v. once weekly) (D). Thermal hyperalgesia (E) and changes in digital nerve maximal action potential amplitudes (F) after 4 doses of paclitaxel (70 $\mathrm{mg} / \mathrm{kg}$, i.v.). All data represent mean values (bars) and SD (error bars) for disposition or SEM for toxicity, using $n=4$ (disposition) or $n=4-24$ (toxicity) per group. ${ }^{*}$ Denotes significant differences from baseline and OATP1B2 ${ }^{-/-}$mice $(P<0.05)$, as evaluated with an unpaired 2 -sided Student's $t$ test with Welch's correction or a 1-way analysis of variance with a Dunnett post-hoc test (B).

paclitaxel, persisted for a week, and then returned to baseline values by 2 weeks. This phenotype is likely causally related to paclitaxel itself, since its main liver metabolites $6 \alpha$-hydroxy-paclitaxel, $3^{\prime}$-p-hydroxy-paclitaxel, and $6 \alpha, 3^{\prime}$-p-dihydroxy-paclitaxel (21) were found to have significantly diminished potential to alter sensitivity to mechanical stimuli compared with the parent drug (Supplemental Figure 1).

In contrast to wild-type mice, OATP1B2-/- mice exposed to paclitaxel showed no significant change in sensitivity to mechanical allodynia (Figure $2 \mathrm{C}$ ). These results suggest that $O A T P 1 B 2^{-/}$mice are protected from acute paclitaxel-induced neuropathy similar to that observed in animals receiving only the paclitaxel vehicle. Similar observations were made with paclitaxel administered over a 4-week period (Figure 2D). Following this chronic regimen, we found that $O A T P 1 B 2^{--}$mice were also protected against paclitaxelinduced thermal hyperalgesia (Figure 2E) and changes in digital nerve maximal action potential amplitude (AMP) (Figure 2F). In support of a functional involvement of OATP1B2 in the paclitaxelinduced toxicity, it is noteworthy to point out that the taxane cabazitaxel, the usage of which is very rarely associated with peripheral neuropathy (22), is not transported by OATP1B-type carriers (18).

Identification of nilotinib as an inhibitor of OATP1B-type transporters. Because several members of the class of TKIs were previously found to potently inhibit OATP1B1 function in vitro
(23), we performed a screen with FDA-approved TKIs and confirmed that several of these agents inhibited OATP1B1 function by at least $75 \%$ using 8-(2-[fluoresceinyl]-aminoethylthio)adenosine-3', $5^{\prime}$-cyclic monophosphate (8Fc-A) (Figure $3 \mathrm{~A}$ ) or estradiol-17 $\beta$-D-glucuronide (E2G) (Figure 3B) as prototypical test substrates. Nilotinib, an inhibitor of the Bcr-Abl tyrosine kinase used for the treatment of patients with chronic myeloid leukemia, was the most potent inhibitor of OATP1B1, in line with several recent reports $(23,24)$. Nilotinib was found to influence OATP1B1 function at concentrations that can be achieved clinically ( $\mathrm{IC}_{50}, \sim 1 \mu \mathrm{M}$ ) (Figure $3 \mathrm{C}$ ), and was also able to inhibit OATP1B3 (Figure 3D). Since nilotinib is itself a poor substrate of OATP1B1 and OATP1B3 (25), the observed interactions are most likely the result of a noncompetitive inhibitory mechanism (Supplemental Figure 2). As anticipated based on the high degree of sequence homology between human and rodent OATP1B-type transporters, their similarity in membrane localization, and largely overlapping substrate and inhibitor specificity (26), we found that nilotinib was also able to inhibit the function of OATP1B2 (Figure 3E). In addition, we confirmed that the plasma levels of paclitaxel were not affected by pretreatment with nilotinib (Figure $3 \mathrm{~F}$ ), suggesting that nilotinib is unlikely to affect paclitaxel-related neurotoxicity phenotypes due to changes in systemic clearance (Supplemental Table 3). 
A

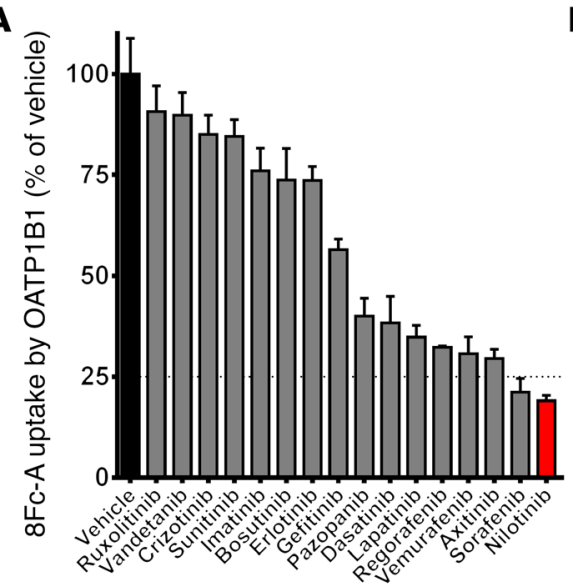

B

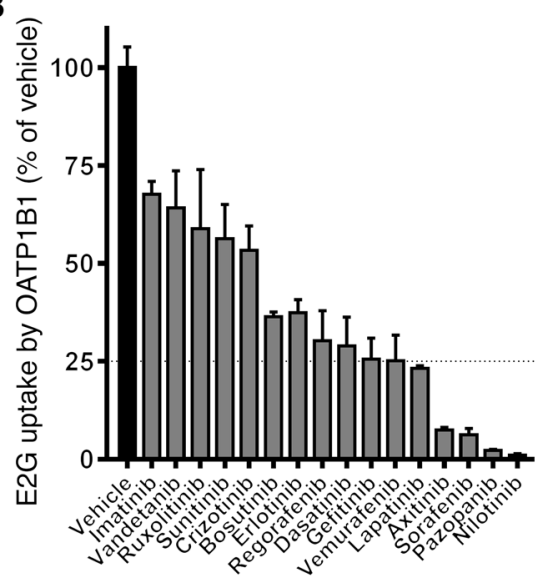

C

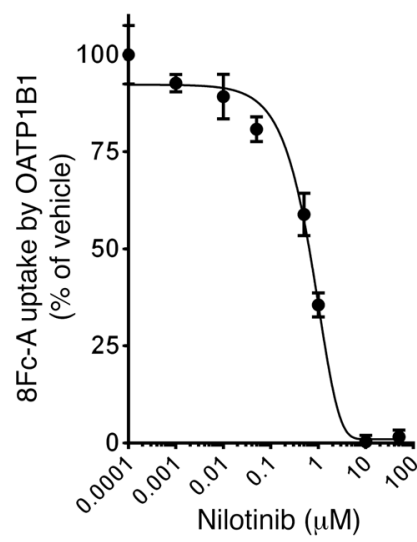

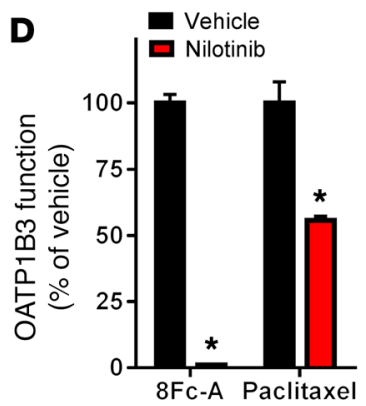
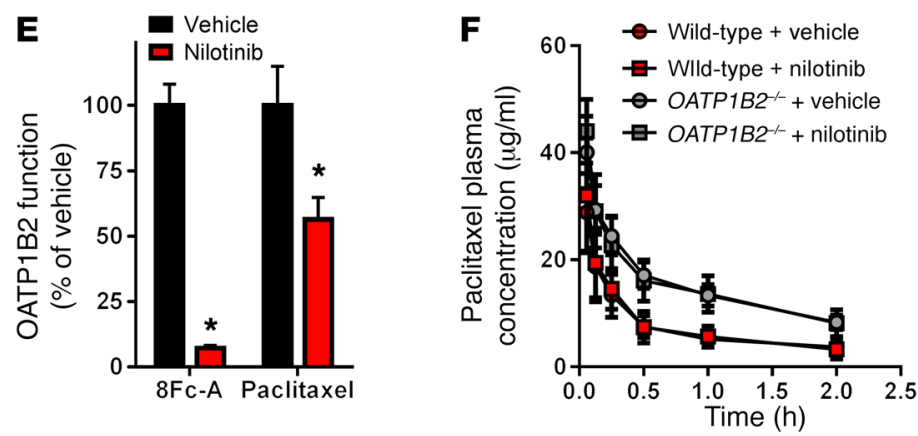

Figure 3. Inhibition of OATP1B-type transporters by nilotinib. Inhibition of OATP1B1 function by tyrosine kinase inhibitors (TKIs) in vitro (10 $\mu$ M; 15 -minute preincubation), using 8-(2-[fluoresceinyl]-aminoethylthio)-adenosine-3',5'-cyclic monophosphate (8Fc-A) (A) and estradiol-17ß-D-glucuronide (E2G) (B) as OATP1B1 substrates in transfected HEK293 cells. (C) Concentration-dependent inhibition of OATP1B1 function by nilotinib using 8Fc-A (2 $\mu$ M; 15 -minute uptake) as a substrate. Inhibition of OATP1B3 (D) and OATP1B2 (E) by nilotinib using 2 different substrates $(2 \mu$ M; $15-$ minute uptake). Data $(n=6-9$ per group) were normalized to substrate uptake in the absence of nilotinib, and corrected for nonspecific uptake in cells transfected with an empty vector. (F) Plasma concentration-time curves of paclitaxel after paclitaxel administration (10 mg/kg) pretreated with nilotinib $(100 \mathrm{mg} / \mathrm{kg} ; \mathrm{p} .0$.) or vehicle $(n=5$ per group). All data represent mean values (bars or symbols) and SD (error bars). ${ }^{*}$ Indicates significant differences from the corresponding vehicle control group $(P<0.05)$, as evaluated with an unpaired 2-sided Student's $t$ test with Welch's correction.

Nilotinib protects against paclitaxel-induced neuropathy. Next, we found that pretreatment of mice with oral nilotinib protected against acute and chronic forms of paclitaxel-induced mechanical allodynia in wild-type mice, and that nilotinib administration had no effect in $O A T P 1 B 2^{--}$mice (Figure 4, A and B). Partial protection against paclitaxel-induced mechanical allodynia could also be afforded by rifampin, a known inhibitor of OATP1B2 (27), and by pazopanib, another TKI identified from our screen, but not sorafenib, a TKI that does not inhibit OATP1B2 in vivo (23) (Supplemental Figure 3). Similarly to the acute toxicities, nilotinib was found to effectively reverse paclitaxel-associated changes in thermal hyperalgesia (Figure 4C) and digital AMP (Figure 4D). Consistent with these observations, we found that administration of nilotinib to mice could inhibit the phosphorylation of the kinase c-Abl, a known target of nilotinib, in both liver and DRG (Supplemental Figure 4). These experiments have not only provided the initiating molecular mechanism responsible for the pathogenesis of paclitaxel-induced peripheral neuropathy, but have also provided proof of principle that targeting of OATP1B-type transporters could be an effective neuroprotective strategy (Figure 5).

Nilotinib as an adjunct to paclitaxel therapy. Although combining paclitaxel with nilotinib could possibly reduce the incidence and severity of drug-induced toxicity, it is important to establish that the anticancer efficacy of paclitaxel is not compromised by nilotinib. The success of such a combination therapy would depend on the selected dosing/scheduling strategy and on the expression status of OATP1B-type transporters in cancer cells. To gain preliminary insights, we evaluated the transcriptional profiles of the OATP1B1 and OATP1B3 genes in human tumor specimens using normalized RNA sequencing data from 29 individual cancer cohorts from The Cancer Genome Atlas (TCGA). This analysis indicated that the transporters are expressed at low levels in samples associated with the main paclitaxel indications such as MBC (Supplemental Figure 5). In agreement with previous studies (28), we were unable to detect appreciable levels of OATP1B1 and OATP1B3 in 48 additional human MBC samples, irrespective of disease stage or receptor status (Supplemental Table 4). This suggests that OATP1B1 and OATP1B3 represent selective uptake transporters that, when inhibited, may reduce cellular toxicity without altering the treatment efficacy of paclitaxel against tumors. Consistent with this supposition, we found that the cellular uptake and cytotoxicity of paclitaxel was not substantially altered by nilotinib in MBC cell lines (Table 1 and Supplemental Figure 6). These results suggest that paclitaxel can be taken up into breast cancer cells by one or more distinct carriers independently of OATP1B1 or OATP1B3, and that these presently unknown carriers are insensitive to nilotinib-mediated inhibition. 
A
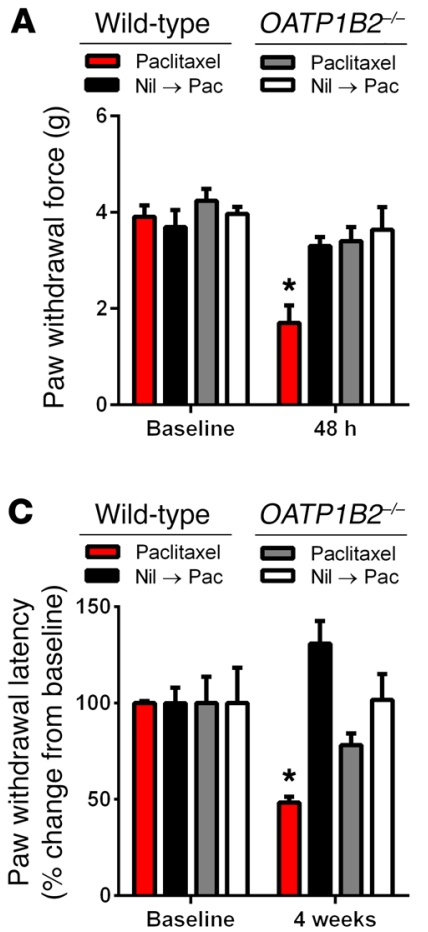

B
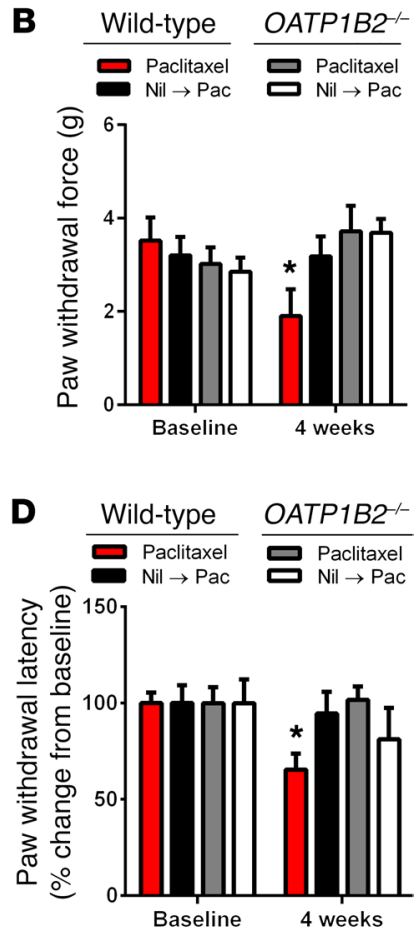

Figure 4. Influence of nilotinib on paclitaxel-induced neurotoxicity. Mechanical allodynia after 1 dose of paclitaxel (Pac; $10 \mathrm{mg} / \mathrm{kg}$, i.v.) (A), or 4 doses (70 $\mathrm{mg} / \mathrm{kg}$, i.v. once weekly) (B) in the presence or absence of pretreatment with nilotinib (Nil; $100 \mathrm{mg} / \mathrm{kg}$; p.o.). Thermal hyperalgesia (C) and changes in digital nerve maximal action potential amplitudes (D) after 4 doses of paclitaxel with or without nilotinib pretreatment. All data represent mean values (bars) and SEM (error bars), using $n=4-24$ per group. *Denotes significant differences from other groups $(P<0.05)$, as evaluated with a 1-way analysis of variance with a Dunnett post-hoc test.

\section{Discussion}

The current study identified OATP1B-type organic anion transporters as important, previously unrecognized contributors to paclitaxel-induced damage to the peripheral nervous system. In particular, our results show that mice with a deficiency of the murine ortholog transporter OATP1B2 are partially protected from acute and chronic paclitaxel-induced neuropathy. In addition, we found that nilotinib, an FDA-approved TKI for the treatment of certain leukemias, is a potent inhibitor of OATP1B-type transport, and that concomitant use of nilotinib with paclitaxel abrogates peripheral neuropathy by inhibiting this drug transport mechanism.

We originally hypothesized that paclitaxel-induced peripheral neuropathy is initiated by extensive uptake of paclitaxel in the nervous system. This idea is supported by the finding that paclitaxel has easy access to DRG where it extensively accumulates, and that detectable levels of paclitaxel can be observed in the sciatic nerve and spinal cord, presumably owing to transport along the centrifugal and centripetal branches of the DRG neuronal axons (8). Moreover, previous studies have demonstrated that the cellular uptake of paclitaxel and structurally related taxanes such as docetaxel occurs via a facilitated transport mechanism (16). Consequently, distribution patterns and pathological changes following paclitaxel administration are restricted to cells in organs capable of transporting paclitaxel from the blood into cells. Indeed, transmembrane transport of taxanes is now widely acknowledged to be mediated by specific OATPs. In particular, we previously reported that paclitaxel and docetaxel are transported substrates of human OATP1B1 (SLCO1B1) and OATP1B3 (SLCO1B3) (14-17), as well as the single functional homolog OATP1B2 (Slco1b2) in mice (18) and rats $(18,29)$. These findings have been independently verified (30-34), and are consistent with in vitro studies that have identified paclitaxel as a potent inhibitor of OATP1B1- $(35,36)$ and OATP1B3mediated transport $(35,37,38)$. However, none of the other known 9 human OATPs or other rodent OATPs are capable of transporting paclitaxel (39), and similar results have been obtained with docetaxel (40). These studies highlight the notion that OATPs capable of transporting paclitaxel need to be expressed in tissues such that the drug can exert cellular injury. We previously reported that OATP1B2 is detectable in mouse DRG (41), and an independent study evaluating all 15 mouse OATPs confirmed expression of OATP1B2 in the membrane of perikarya and neurites of cultured primary murine neurons (42). Our present identification of high OATP1B2 protein expression in DRG is thus consistent with these prior observations.

The demonstration that OATP1B2 plays an important role in a clinically relevant paclitaxel-related toxicity provides a compelling rationale for the development of translational interventions for paclitaxel-containing regimens in patients involving the use of specific inhibitors of these carriers. Such agents would ideally have high potency, high specificity, low drug-drug interaction potential, intrinsic antitumor properties, favorable pharmaceutical properties, and nonoverlapping toxicity profiles. We hypothesized that the class of TKIs is of particular interest in this context, as these agents have many of the above features, and several members of the class have been found to potently inhibit various drug transporters (43). The ultimate identification of nilotinib as an inhibitor of OATP1B2, without being itself a transported substrate, is consistent with a recent report suggesting
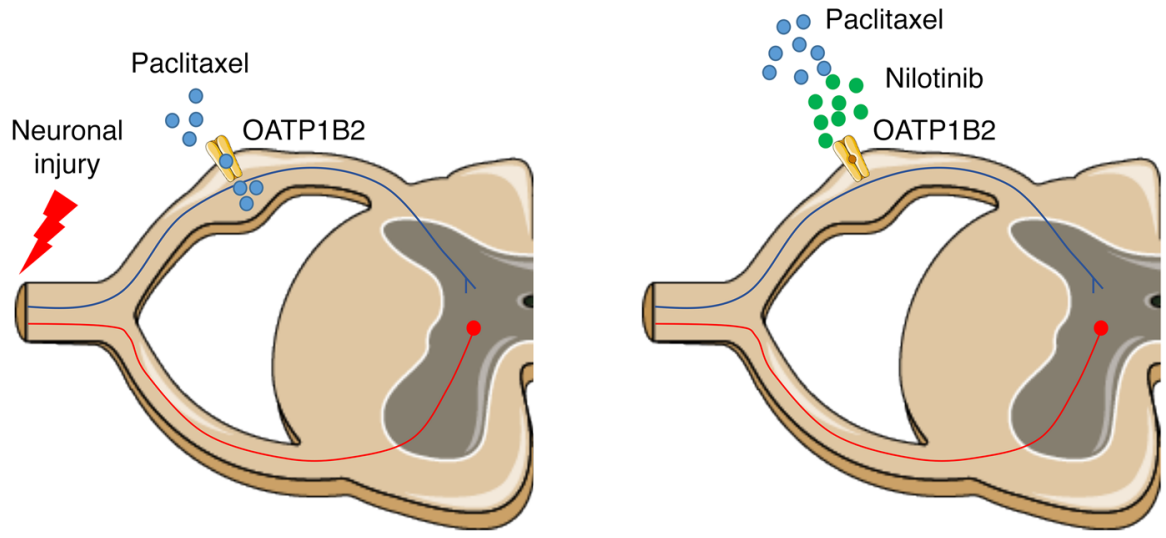

Figure 5. Proposed model of paclitaxel-induced injury to the peripheral nervous system. Paclitaxel enters the nervous system through OATP1B2, ultimately leading to peripheral neuropathy (left), and these effects can be prevented by the OATP1B2 inhibitor nilotinib (right). 
Table 1. Influence of nilotinib on paclitaxel activity against breast cancer cells ${ }^{A}$

\begin{tabular}{|c|c|c|c|c|c|}
\hline \multicolumn{2}{|l|}{ Cell line } & Paclitaxel & Nilotinib & $\mathrm{Pac}+\operatorname{Nil}(1 \mu \mathrm{M})$ & $\mathrm{Pac}+\mathrm{Nil}(5 \mu \mathrm{M})$ \\
\hline \multirow[t]{2}{*}{ MDA-MB-468 } & $\mathrm{IC}_{50}(\mathrm{nM})$ & 22.8 & 4,830 & 9.06 & 4.37 \\
\hline & Uptake (pmol/mg) & $13.4 \pm 1.40$ & $\mathrm{~N} / \mathrm{A}$ & $\mathrm{N} / \mathrm{A}$ & $9.86 \pm 3.24$ \\
\hline \multirow[t]{2}{*}{ MCF-7 } & $\mathbb{I C}_{50}(\mathrm{nM})$ & 157 & 10,200 & 61.9 & 4.70 \\
\hline & Uptake (pmol/mg) & $6.04 \pm 1.09$ & $\mathrm{~N} / \mathrm{A}$ & $\mathrm{N} / \mathrm{A}$ & $4.25 \pm 1.11$ \\
\hline \multirow[t]{2}{*}{ T-47D } & $\mathrm{IC}_{50}(\mathrm{nM})$ & 85.9 & 3,710 & 33.9 & $<0.1$ \\
\hline & Uptake (pmol/mg) & $8.96 \pm 0.804$ & $\mathrm{~N} / \mathrm{A}$ & $\mathrm{N} / \mathrm{A}$ & $6.71 \pm 0.906$ \\
\hline \multirow[t]{2}{*}{ MDA-MB-231 } & $\mathbb{I C}_{50}(\mathrm{nM})$ & 20.9 & 10,900 & 11.9 & 10.4 \\
\hline & Uptake (pmol/mg) & $5.87 \pm 1.93$ & $\mathrm{~N} / \mathrm{A}$ & $\mathrm{N} / \mathrm{A}$ & $4.67 \pm 1.38$ \\
\hline \multirow[t]{2}{*}{ HS-578T } & $\mathrm{IC}_{50}(\mathrm{nM})$ & 26.1 & 3,190 & 28.8 & 13.1 \\
\hline & Uptake (pmol/mg) & $13.6 \pm 6.19$ & $\mathrm{~N} / \mathrm{A}$ & $\mathrm{N} / \mathrm{A}$ & $11.9 \pm 4.77$ \\
\hline
\end{tabular}

${ }^{A}$ All data are presented as mean values and SD, using $n=6-8$ per group. The cell growth inhibitory potential of paclitaxel (range, $0.00001-50 \mu \mathrm{M})$ or nilotinib $(0.01-100 \mu \mathrm{M}$ ) was evaluated with an MTT assay using 72-hour continuous exposure. The cell growth inhibitory potential of paclitaxel (range, $0.01-100 \mu \mathrm{M}$ ) was also evaluated in the presence of nilotinib (1 or $10 \mu \mathrm{M} ; 15$-minute preincubation). Accumulation of paclitaxel ( $2 \mu \mathrm{M} ; 5$-minute uptake) was determined in the presence or absence of nilotinib (15-minute preincubation with $10 \mu \mathrm{M}$ or vehicle). $I_{50}$, half-maximum inhibitory concentration; Pac, paclitaxel; Nil, nilotinib. observation that the TKI does not antagonize the cytotoxic effects of paclitaxel in the same 5 preclinical models of MBC, as determined by an MTT assay in 2-dimensional culture. These findings are in line with previously reported synergistic in vitro effects of nilotinib and paclitaxel in models of ovarian cancer (49) and certain ABCB1-overexpressing tumors (50). Near the completion of our investigations, we became aware of an ongoing project, recently published (51), aimed at identifying promising combinations of approved drugs with potent anticancer activity against a panel of 60 human tumor cell lines for translation to clinical trials. Strikingly, in that study, the nilotinibpaclitaxel combination was identified as an exquisitely active drug pair against breast cancer cell lines, resulting in tumor regressions in the MDAMB-468 xenograft model with no tumor regrowth observed for more than 80 days following the end of therapy (51). Although in vivo confirmation is required in patient-derived xenograft model systems that more faithfully represent the charac-

potent in vitro inhibition by nilotinib of OATP1B-type carriers at concentrations that are predicted to have potential in vivo significance (44). The mechanism by which nilotinib affects OATP1B2 function requires further investigation.

Nilotinib was originally developed as an inhibitor of the BcrAbl tyrosine kinase and has been used clinically as a once/twice daily oral tablet for the treatment of patients who are in the chronic and accelerated phases of Philadelphia-chromosome-positive chronic myeloid leukemia. Although the combination of nilotinib and paclitaxel has not been explored clinically in any disease, nilotinib has pharmaceutical and pharmacological features that suggest it might be an excellent modulator of paclitaxel toxicities. These include good oral absorption, a relatively slow systemic clearance, and a long half-life (45), thus ensuring sufficiently high and persistent local drug levels. Interestingly, high-dose TKI pulse exposure - rather than the more commonly used chronic lowdose schedules involving daily dosing - is becoming an increasingly frequently applied concept in the treatment of cancer (46), and the clinical experience with such a strategy will ultimately allow easy translation of our proposed concept to use nilotinib as a transporter inhibitor in conjunction with paclitaxel-based chemotherapy in patients. We acknowledge that, while most side effects associated with nilotinib are mild, reversible, and easily managed, the drug's prescribing information carries a black box warning for QT prolongation (47). It should be pointed out, however, that the median time from the start of nilotinib therapy using a conventional chronic regimen (i.e., once or twice daily dosing without interruption) to the onset of such $\mathrm{QT}$ events is greater than 14 months (range, 2-68 months) (48). In our studies, we aim to interrogate the response of the peripheral nervous system to the nilotinib-paclitaxel combination following acute or intermittent exposure to the TKI. Therefore, we anticipate that nilotinib will not be intrinsically toxic in such combination therapy regimens.

The translational potential of a nilotinib-based intervention strategy to prevent paclitaxel toxicity is further supported by our teristics of primary human MBC compared with xenografted cell lines (52), these combined initial observations indicate that combining paclitaxel with TKIs such as nilotinib has the potential to simultaneously reduce toxicities and increase anticancer effects.

Collectively, we identified a previously unrecognized pathway of paclitaxel-induced peripheral neurotoxicity that is mediated by OATP1B-type transporters. The function of this transport system can be inhibited by nilotinib through a noncompetitive mechanism, without compromising the anticancer properties of paclitaxel in models of MBC. These findings not only shed light on the etiology of paclitaxel-induced neurotoxicity, but provide a rationale for the future development of new targeted interventions using transporter inhibitors to mitigate a debilitating side effect associated with paclitaxel.

\section{Methods}

In vitro transport studies. Human embryonic kidney (HEK293) cells (ATCC) transfected with OATP1B1, OATP1B3, or OATP1B2 have been described previously (15). The transporter nomenclature used throughout is based on recent recommendations proposed by Hagenbuch and Stieger (53). The transporter-expressing cells were functionally characterized by assessing the uptake of $\mathrm{E} 2 \mathrm{G}(0.1$ or $2 \mu \mathrm{M})$ by OATP1B1, OATP1B3, and OATP1B2, and estrone-3-sulfate ( 2 or $2.5 \mu \mathrm{M}$ ) by OATP2B1. HEK293 cells overexpressing OATP2B1 were created by transfecting an empty pCMV6-entry vector or a construct containing OATP2B1 cDNA using Lipofectamine 3000 (Thermo Fisher Scientific), and cells were selected based on G418 resistance. Overexpression of transporters was confirmed using TaqMan probes (Applied Biosystems). HEK293 cells were maintained in DMEM supplemented with $10 \%$ heat-inactivated FBS, and routinely checked to ensure there was no mycoplasma contamination (MycoAlert Detection Kit). Passages were kept to a minimum and cells were not used beyond passage 30 .

The cell culture conditions and details of accumulation experiments for $\left[{ }^{3} \mathrm{H}\right]$ paclitaxel (specific activity, $25.6 \mathrm{Ci} / \mathrm{mmol}$; Vitrax) were performed as described previously (15). Radioactivity was quantified 
by liquid scintillation counting using an LS 6500 Counter (Beckman). Drug uptake results were normalized to total protein content and then to data obtained in cells carrying an empty vector plasmid, which was set to $100 \%$. Preliminary experiments indicated that phenol red, a $\mathrm{pH}$ indicator in trypsin used to resuspend cultured cells, influenced OATP1B-mediated cellular uptake of taxanes, and therefore these studies were performed in phenol red-free conditions. The influence of TKIs (10 $\mu \mathrm{M}$; 15-minute preincubation) on OATP1B1, OATP1B3, or OATP1B2 function was evaluated using E2G or 8Fc-A (Axxora LLC) as prototypical substrates.

Murine pharmacokinetic studies. Wild-type and OATP1B2-/- mice, both on a DBA/1LacJ background and between 8 and 12 weeks of age, were housed in a temperature-controlled environment with a 12-hour light/dark cycle. These mice were provided by Richard B. Kim (Western University, London, Ontario, Canada) and Jeffrey L. Stock (Pfizer Inc., Groton, Connecticut, USA). All mice received a standard diet and water ad libitum. For pharmacokinetic studies, paclitaxel was formulated in Cremophor EL/ethanol (1:1, v/v; Taxol; Bristol-Myers Squibb). These solutions were diluted in normal saline and administered by i.v. bolus in the tail vein at a dose of $10 \mathrm{mg} / \mathrm{kg}$. In select experiments, oral nilotinib (formulated in $0.5 \%$ hydroxypropyl-methylcellulose in water) was administered 30 minutes before paclitaxel at a dose of $100 \mathrm{mg} / \mathrm{kg}$. At select time points after paclitaxel administration, blood samples (30 $\mu \mathrm{l}$ each) were taken from individual mice at 3.5, 7.5, and 15 minutes from the submandibular vein using a lancet, and at 30 and 60 minutes from the retro-orbital venous plexus using a capillary. The total blood volume collected during the procedure from each mouse was $150 \mu \mathrm{l}$. A final additional blood draw was obtained at 120 minutes by cardiac puncture using a syringe and needle. Isoflurane was used as an anesthetic. All blood samples were centrifuged at $1,500 \mathrm{~g}$ for 5 minutes, and plasma was separated and stored at $-80^{\circ} \mathrm{C}$ until analysis by a validated method based on reversed-phase liquid chromatography coupled to tandem mass-spectrometric detection (LC/MS/MS) using a previously published procedure (54), with modifications (see below).

Livers and DRG were collected from the same animals at $120 \mathrm{~min}$ utes and, to prevent continuing metabolic activity, tissues were snapfrozen using liquid nitrogen. All tissue specimens were stored at $-80^{\circ} \mathrm{C}$ until further processing, as described previously (15). All organs were diluted in blank (drug-free) human plasma $(1: 4, w / v)$ in a 2-ml Eppendorf vial. Next, a 5-mm stainless steel bead (Qiagen) was added, and the samples were homogenized with a TissueLyser (Qiagen), and processed for 4 minutes at $40 \mathrm{~Hz}$. Finally, the beads were removed and the homogenized samples were stored at $-70^{\circ} \mathrm{C}$ until analysis by LC/ MS/MS. Noncompartmental pharmacokinetic parameters were calculated using WinNonlin 6.2 software (Pharsight), and drug levels in tissues were corrected for contaminating plasma.

Determination of drug levels. Paclitaxel was quantified using LC/ MS/MS. Sample extracts were injected into an Alltima HP C18 HL $3-\mu \mathrm{m}$ column $(50 \times 2.1 \mathrm{~mm}$ internal diameter, Alltech Applied Science) by a Waters 2795 Separation Module. The mobile phase for determination of paclitaxel was composed of acetonitrile and water containing formic acid $(0.1 \% \mathrm{v} / \mathrm{v})$, and was delivered using linear gradient settings at a flow rate of $0.2 \mathrm{ml} / \mathrm{min}$. Detection was performed with a MicroMass Quatro Micro triple-quadrupole mass spectrometer (Waters) in the positive ion mode. The electrospray ionization was set at $3.8 \mathrm{kV}$ and the cone voltage at $18 \mathrm{~V}$. The dwell times were set at $150 \mathrm{~ms}$ and the interchannel delay at $50 \mathrm{~ms}$. A multiple-reaction monitoring (MRM) mode was applied for the quantification with the following parameters: $m / z 854 \rightarrow 286$, collision energy at $20 \mathrm{eV}$ for paclitaxel; and $m / z 859 \rightarrow 291$, collision energy at $20 \mathrm{eV}$ for the internal standard, paclitaxel-d5. The collision cell pressure was set at approximately $4 \times 10^{-3} \mathrm{mbar}$ (argon). Samples for the quantification of paclitaxel were prepared by extraction of $100-\mu$ l aliquots with $200 \mu \mathrm{l}$ of an acetonitrile solution of the internal standard, and $1 \mathrm{ml}$ of $n$-butylchloride. After vigorous mixing and centrifugation for 10 minutes at $18,000 \mathrm{~g}$, the clear supernatant was evaporated at a temperature of $70^{\circ} \mathrm{C}$. The residue was dissolved in $150 \mu \mathrm{l}$ of a mixture of acetonitrile/water/formic acid (40:60:0.1, v/v/v), from which aliquots of $5 \mu \mathrm{l}$ were injected into the LC/MS/MS system. The lower limit of quantification (LLQ) for paclitaxel was determined to be $20.0 \mathrm{ng} / \mathrm{ml}$, and the calibration curve ranged from 20.0 to $1,000 \mathrm{ng} /$ $\mathrm{ml}$. The within-run and between-run precisions were within $7.14 \%$, while the accuracy ranged from $88.5 \%$ to $94.1 \%$.

Anesthesia. For the neurophysiological recordings, anesthesia was induced with $3 \%$ isoflurane carried in oxygen followed by $1 \%$ isoflurane through a nose cone for maintenance throughout the procedures. This was adequate to suppress the corneal blink response and any withdrawal response to a noxious stimulus. The body temperature was maintained at $37^{\circ} \mathrm{C}$ using a heating pad (ATC-2000, World Precision Instruments) to minimize isoflurane-induced hypothermia.

Experimental design of neurotoxicity studies. The wild-type and $O A T P 1 B 2^{-1-}$ mice were randomly divided into 2 groups based on baseline neurophysiology results (see below); one group was treated with paclitaxel only, and the other group was pretreated with nilotinib, 30 minutes before paclitaxel. Mice were examined daily for sickness symptoms due to drug treatment. Changes in their appearance (e.g., kyphosis and altered grooming), behavior (e.g., altered nesting), and/ or activity (e.g., altered exploring) were monitored. Body weight was recorded before treatment and at least once a week for general toxicity assessment and drug dose adjustment if indicated. Acute toxicities were evaluated following a single i.v. dose of paclitaxel $(10 \mathrm{mg} / \mathrm{kg})$, formulated in Cremophor EL/ethanol $(1: 1, \mathrm{v} / \mathrm{v})$. In a separate set of experiments, acute toxicities were evaluated following a single i.v. dose of the paclitaxel metabolites $6 \alpha$-hydroxy-paclitaxel (Toronto Research Chemicals; catalog H948890), 3'-p-hydroxy-paclitaxel (Toronto Research Chemicals; catalog H948895), or 6 $\alpha, 3^{\prime}-p$-dihydroxypaclitaxel (Toronto Research Chemicals; catalog D454200), all formulated in Cremophor EL/ethanol $(1: 1, \mathrm{v} / \mathrm{v})$ and administered at a dose of $10 \mathrm{mg} / \mathrm{kg}$. In select experiments, single doses of nilotinib (100 $\mathrm{mg} / \mathrm{kg}$; per os [p.o.]), rifampin (20 mg/kg; i.p.), pazopanib (20 mg/ $\mathrm{kg}$; p.o.), or sorafenib (20 mg/kg; p.o.) were administered 30 minutes before the administration of paclitaxel.

For chronic toxicities, paclitaxel was administered once every week for 4 weeks at an i.v. dose of paclitaxel, formulated in a mixture of polysorbate $80 /$ ethanol (1:1, v/v), preceded by nilotinib $(100 \mathrm{mg} /$ $\mathrm{kg}$; p.o.) or its vehicle (0.5\% methylcellulose in water). Preliminary dose-response studies $(60,70$, or $80 \mathrm{mg} / \mathrm{kg})$ indicated that a weekly paclitaxel dose of $70 \mathrm{mg} / \mathrm{kg}$ in wild-type DBA/1LacJ mice produced neuropathy phenotypes, in particular decreases in the distal maximal action potential amplitudes (AMP) but not in digital nerve conductance velocity (NCV) (Supplemental Figure 7), that were quantitatively and qualitatively similar to those reported previously in other mouse strains such as nude-Foxn $1^{\text {nu }}(4)$. 
Neurophysiological analyses. Nerve conduction studies were performed as previously described using a clinical electrodiagnostic system (Ultra Pro S100, Natus Neurology) (4). Briefly, 2 needle electrodes (Natus Neurology) were placed near the fourth digit to stimulate the digital nerve, and 2 recording electrodes (ADD Company) were placed $10 \mathrm{~mm}$ proximally. A total of 10 supramaximal stimulations were delivered, and the peak-to-peak compound AMP was obtained and averaged. All the neurophysiological recordings were performed with mice under isoflurane anesthesia delivered via nose cone in a temperature/humidity controlled room. Body temperature was continuously monitored and maintained during the recordings. The baseline recordings were measured before starting the drug treatments in order to randomize animals into homogeneous groups. Because of interexperimental variability in baseline readings of AMP and NCV across the various studies, these parameters were always normalized to a value of $100 \%$.

Mechanical threshold assessments. The nocifensive behaviors (hind paw flinching) after mechanical stimulation (Von Frey test) (41) was used to assess the development of mechanical allodynia, as previously described (4). A servo-controlled mechanical stimulator with a blunt metallic filament $(0.5 \mathrm{~mm}$ diameter) was positioned under the surface of the hind paw to exert a progressively increasing punctate pressure with a gram force ramp of $1 \mathrm{~g} / \mathrm{s}$. Thermal hyperalgesia (Hargreaves test) was evaluated with a dynamic esthesiometer (model 390, IITC Life Science) (55). On the day of testing, mice were placed in a Plexiglas chamber for 45 minutes to acclimate. When a spontaneous hind paw withdrawal occurred, the stimulus was automatically stopped and the gram force of the pressure or the time to withdrawal was recorded. This was assessed alternately on each hind paw every 2 minutes for 3 consecutive trials to obtain a mean value of the maximal pressure (expressed in grams) or heat tolerated by the mice. To prevent tissue damage, an upper cutoff limit of $15 \mathrm{~g}$ or 20 seconds was set, after which the increasing mechanical or thermal stimulus was terminated.

Evaluation of antitumor efficacy. Accumulation of paclitaxel levels in breast cancer cell lines was determined as described above for transfected HEK293 cells at a concentration of $2 \mu \mathrm{M}$ (5-minute uptake) in the presence or absence of nilotinib (15-minute preincubation with $10 \mu \mathrm{M}$ or vehicle). For in vitro uptake and cytotoxicity studies, stock solutions of nilotinib and paclitaxel were prepared with the drugs dissolved in DMSO. The cell growth inhibitory potential was evaluated with an MTT assay using 72-hour continuous exposure. The influence of nilotinib ( 1 or $10 \mu \mathrm{M}$ or vehicle; 15-minute preincubation) on the cytotoxicity of paclitaxel (range, $0.00001-50 \mu \mathrm{M})$ was evaluated in the replicating breast cancer cell lines MDA-MB-468, MCF-7, T-47D, MDA-MB-231, and HS-578T (National Cancer Institute, NIH).

Immunohistochemistry and immunoblotting. Protein expression analyses on isolated liver and DRG samples from wild-type and $O A T$ $P 1 B 2^{-/}$mice were performed as described previously (41). For immunohistochemistry, a previously validated OATP1B2 primary antibody was provided by Curtis D. Klaassen and Iván L. Csanaky (University of Kansas Medical Center, Kansas City, Kansas, USA), and Alexa Fluor 647 goat anti-rabbit IgG (H+L) (Invitrogen, A21244, lot 1871168) was used as a secondary antibody. For immunoblotting, the same primary antibody was used and peroxidase-conjugated AffiniPure rabbit antimouse IgG $(\mathrm{H}+\mathrm{L})$ (Jackson ImmunoResearch, catalog 315-035-045, lot 119936) as a secondary antibody, and the procedure was validated on liver samples (Supplemental Figure 8). The antibodies for phospho-c-Abl (Tyr245) and total c-Abl were obtained from Cell Signaling Technology (catalog 07-787 and 2862, respectively).

Real-time PCR. Expression of transporter genes was analyzed with $\mathrm{RT}^{2}$ Profiler PCR arrays (SABiosciences), as described previously (56). Gene expression analyses in human tumors were based on the Pan Cancer gene expression data set (57), and extracted using the UCSC Xena browser. Tissue plates containing cDNA from 48 human breast cancer tissues (Tissue Scan Arrays BCRT-303) were obtained from Origene. RNA was reverse transcribed using SuperScript III FirstStrand Synthesis SuperMix for Real-Time PCR (Invitrogen), according to the manufacturer's recommendations. Gene transcripts were quantified using TaqMan Universal PCR Master Mix (Thermo Fisher Scientific) and primers obtained from Origene that were specific for OATP1B1 (HP209396) and OATP1B3 (HP213461). Reactions were carried out in triplicate as previously reported (41). Transcripts of each sample were normalized to the housekeeping gene, GAPDH.

Statistics. All data are presented as mean values and SD, unless stated otherwise. All experiments were performed independently on at least 2 separate occasions. An unpaired 2-sided Student's $t$ test with Welch's correction or 1-way analysis of variance with a Dunnett posthoc test was used to evaluate statistical significance, using $P$ less than 0.05 as the cutoff.

Study approval. All animals were housed and handled in accordance with the Institutional Animal Care and Use Committee of The Ohio State University, and all studies were performed in accordance with national animal protection laws.

\section{Author contributions}

AFL, GC, AS, and SH conceived and designed the experiments. AFL, JAS, AAG, KWH, MSP, MC, KMH, VC, OC, TF, and SH conducted the in vitro experiments. PDB performed analytical assays. AFL, PA, AC, WDA, and SH provided reagents, generated cell culture models, and performed in vivo experiments. AFL, AS, and SH performed the statistical analyses. AFL, JAS, RHM, RER, MBL, LESC, GC, AS, and SH analyzed the data. AFL, AS, and SH wrote the manuscript. All authors contributed to editing the manuscript.

\section{Acknowledgments}

We thank Navjot Pabla, Tore Stage, and Deanna Kroetz for helpful scientific discussion and critical review of the manuscript. The results shown are in part based on data generated by the TCGA Research Network (http://cancergenome.nih.gov). The project was supported in part by NIH grants R01CA187176 and R01CA215802, and by the OSU Comprehensive Cancer Center using Pelotonia funds. Any opinions, findings, and conclusions expressed in this material are those of the authors and do not necessarily reflect those of the Pelotonia program. The funding bodies had no role in the preparation of the manuscript.

Address correspondence to: Alex Sparreboom, Division of Pharmaceutics, College of Pharmacy, The Ohio State University, 500 W. 12th Avenue, Columbus, Ohio 43210, USA. Phone: 614.685.6014; Email: sparreboom.1@osu.edu. Or to: Shuiying Hu, Division of Pharmaceutics, College of Pharmacy, The Ohio State University, 460 W. 12th Avenue, Columbus, Ohio 43210, USA. Phone: 614.685.7551; Email: hu.1333@osu.edu. 
1. De Iuliis F, Taglieri L, Salerno G, Lanza R, Scarpa $\mathrm{S}$. Taxane induced neuropathy in patients affected by breast cancer: Literature review. Crit Rev Oncol Hematol. 2015;96(1):34-45.

2. Peters CM, et al. Intravenous paclitaxel administration in the rat induces a peripheral sensory neuropathy characterized by macrophage infiltration and injury to sensory neurons and their supporting cells. Exp Neurol. 2007;203(1):42-54.

3. Mielke S, Sparreboom A, Mross K. Peripheral neuropathy: a persisting challenge in paclitaxelbased regimes. Eur J Cancer. 2006;42(1):24-30.

4. Carozzi VA, Canta A, Chiorazzi A. Chemotherapy-induced peripheral neuropathy: What do we know about mechanisms? Neurosci Lett. 2015;596:90-107.

5. Brewer JR, Morrison G, Dolan ME, Fleming GF. Chemotherapy-induced peripheral neuropathy: Current status and progress. Gynecol Oncol. 2016;140(1):176-183.

6. Marmiroli P, Cavaletti G. Drugs for the treatment of peripheral neuropathies. Expert Opin Pharmacother. 2016;17(3):381-394.

7. Yan X, et al. Paclitaxel induces acute pain via directly activating toll like receptor 4. Mol Pain. 2015;11:10.

8. Cavaletti G, et al. Distribution of paclitaxel within the nervous system of the rat after repeated intravenous administration. Neurotoxicology. 2000;21(3):389-393.

9. Loprinzi CL, et al. Natural history of paclitaxelassociated acute pain syndrome: prospective cohort study NCCTG N08C1. JClin Oncol. 2011;29(11):1472-1478.

10. Scripture CD, Figg WD, Sparreboom A. Peripheral neuropathy induced by paclitaxel: recent insights and future perspectives. Curr Neuropharmacol. 2006;4(2):165-172.

11. Schneider BP, Hershman DL, Loprinzi C. Symptoms: chemotherapy-induced peripheral neuropathy. Adv Exp Med Biol. 2015;862:77-87.

12. Schneider BP, et al. Genome-wide association studies for taxane-induced peripheral neuropathy in ECOG-5103 and ECOG-1199. Clin Cancer Res. 2015;21(22):5082-5091.

13. Hershman DL, et al. Prevention and management of chemotherapy-induced peripheral neuropathy in survivors of adult cancers: American Society of Clinical Oncology clinical practice guideline. J Clin Oncol. 2014;32(18):1941-1967.

14. Baker SD, et al. Pharmacogenetic pathway analysis of docetaxel elimination. Clin Pharmacol Ther. 2009;85(2):155-163.

15. de Graan AJ, et al. Influence of polymorphic OAT P1B-type carriers on the disposition of docetaxel. Clin Cancer Res. 2012;18(16):4433-4440.

16. Smith NF, Acharya MR, Desai N, Figg WD, Sparreboom A. Identification of OATP1B3 as a highaffinity hepatocellular transporter of paclitaxel. Cancer Biol Ther. 2005;4(8):815-818.

17. Smith NF, et al. Variants in the SLCO1B3 gene: interethnic distribution and association with paclitaxel pharmacokinetics. Clin Pharmacol Ther. 2007;81(1):76-82.

18. Nieuweboer AJ, et al. Influence of drug formulation on OATP1B-mediated transport of paclitaxel. Cancer Res. 2014;74(11):3137-3145.

19. Durmus S, Lozano-Mena G, van Esch A, Wage- naar E, van Tellingen O, Schinkel AH. Preclinical mouse models to study human OATP1B1- and OATP1B3-mediated drug-drug interactions in vivo. Mol Pharm. 2015;12(12):4259-4269.

20. Boehmerle W, Huehnchen P, Peruzzaro S, Balkaya M, Endres M. Electrophysiological, behavioral and histological characterization of paclitaxel, cisplatin, vincristine and bortezomibinduced neuropathy in C57BL/6 mice. Sci Rep. 2014;4:6370.

21. Sparreboom A, Huizing MT, Boesen JJ, Nooijen WJ, van Tellingen O, Beijnen JH. Isolation, purification, and biological activity of mono- and dihydroxylated paclitaxel metabolites from human feces. Cancer Chemother Pharmacol. 1995;36(4):299-304.

22. Omlin A, et al. Analysis of side effect profile of alopecia, nail changes, peripheral neuropathy, and dysgeusia in prostate cancer patients treated with docetaxel and cabazitaxel. Clin Genitourin Cancer. 2015;13(4):e205-e208.

23. Hu S, Mathijssen RH, de Bruijn P, Baker SD, Sparreboom A. Inhibition of OATP1B1 by tyrosine kinase inhibitors: in vitro-in vivo correlations. $\mathrm{Br}$ J Cancer. 2014;110(4):894-898.

24. Khurana V, Minocha M, Pal D, Mitra AK. Role of OATP-1B1 and/or OATP-1B3 in hepatic disposition of tyrosine kinase inhibitors. Drug Metabol Drug Interact. 2014;29(3):179-190.

25. Zimmerman EI, et al. Contribution of OATP1B1 and OATP1B3 to the disposition of sorafenib and sorafenib-glucuronide. Clin Cancer Res. 2013;19(6):1458-1466.

26. Roth M, Obaidat A, Hagenbuch B. OATPs, OATs and OCTs: the organic anion and cation transporters of the SLCO and SLC22A gene superfamilies. Br J Pharmacol. 2012;165(5):1260-1287.

27 . Bins S, et al. Influence of OATP1B1 function on the disposition of sorafenib- $\beta$-D-glucuronide. Clin Transl Sci. 2017;10(4):271-279.

28. Lancaster CS, Sprowl JA, Walker AL, Hu S, Gibson AA, Sparreboom A. Modulation of OATP1Btype transporter function alters cellular uptake and disposition of platinum chemotherapeutics. Mol Cancer Ther. 2013;12(8):1537-1544.

29. Franke RM, Carducci MA, Rudek MA, Baker SD, Sparreboom A. Castration-dependent pharmacokinetics of docetaxel in patients with prostate cancer. J Clin Oncol. 2010;28(30):4562-4567.

30. Iusuf D, et al. Human OATP1B1, OATP1B3 and OATP1A2 can mediate the in vivo uptake and clearance of docetaxel. Int J Cancer. 2015;136(1):225-233.

31. Marada VV, Flörl S, Kühne A, Burckhardt G, Hagos Y. Interaction of human organic anion transporter polypeptides 1B1 and 1B3 with antineoplastic compounds. Eur JMed Chem. 2015;92:723-731.

32. Sun $X$, et al. Pharmacokinetic effects of curcumin on docetaxel mediated by OATP1B1, OATP1B3 and CYP450s. Drug Metab Pharmacokinet. 2016;31(4):269-275.

33. van de Steeg E, van Esch A, Wagenaar E, Kenworthy KE, Schinkel AH. Influence of human OATP1B1, OATP1B3, and OATP1A2 on the pharmacokinetics of methotrexate and paclitaxe in humanized transgenic mice. Clin Cancer Res. 2013;19(4):821-832.

34. van de Steeg E, et al. High impact of Oatp1a/1b transporters on in vivo disposition of the hydrophobic anticancer drug paclitaxel. Clin Cancer Res. 2011;17(2):294-301.

35. Gui C, et al. Effect of pregnane X receptor ligands on transport mediated by human OATP1B1 and OATP1B3. Eur J Pharmacol. 2008;584(1):57-65.

36. Gui C, Wahlgren B, Lushington GH, Hagenbuch B. Identification, Ki determination and CoMFA analysis of nuclear receptor ligands as competitive inhibitors of OATP1B1-mediated estradiol17beta-glucuronide transport. Pharmacol Res. 2009;60(1):50-56.

37. Letschert K, Faulstich H, Keller D, Keppler D. Molecular characterization and inhibition of amanitin uptake into human hepatocytes. Toxicol Sci. 2006;91(1):140-149.

38. Yamaguchi H, et al. Rapid screening of antineoplastic candidates for the human organic anion transporter OATP1B3 substrates using fluorescent probes. Cancer Lett. 2008;260(1-2):163-169.

39. Svoboda M, et al. Expression of organic aniontransporting polypeptides 1B1 and 1B3 in ovarian cancer cells: relevance for paclitaxel transport. Biomed Pharmacother. 2011;65(6):417-426.

40. Lee HH, Leake BF, Teft W, Tirona RG, Kim RB, Ho RH. Contribution of hepatic organic aniontransporting polypeptides to docetaxel uptake and clearance. Mol Cancer Ther. 2015;14(4):994-1003.

41. Sprowl JA, et al. Oxaliplatin-induced neurotoxicity is dependent on the organic cation transporter OCT2. Proc Natl Acad Sci USA. 2013;110(27):11199-11204.

42. Feurstein D, Kleinteich J, Heussner AH, Stemmer K, Dietrich DR. Investigation of microcystin congener-dependent uptake into primary murine neurons. Environ Health Perspect. 2010;118(10):1370-1375.

43. Neul C, Schaeffeler E, Sparreboom A, Laufer S, Schwab M, Nies AT. Impact of membrane drug transporters on resistance to small-molecule tyrosine kinase inhibitors. Trends Pharmacol Sci. 2016;37(11):904-932.

44. Khurana V, Minocha M, Pal D, Mitra AK. Inhibition of OATP-1B1 and OATP-1B3 by tyrosine kinase inhibitors. Drug Metabol Drug Interact. 2014;29(4):249-259.

45. Xia B, Heimbach T, He H, Lin TH. Nilotinib preclinical pharmacokinetics and practical application toward clinical projections of oral absorption and systemic availability. Biopharm Drug Dispos. 2012;33(9):536-549.

46. Lipka DB, et al. Intracellular retention of ABL kinase inhibitors determines commitment to apoptosis in CML cells. PLOS ONE. 2012;7(7):e40853.

47. Rios MB, Ault P. Identification of side effects associated with intolerance to BCR-ABL inhibitors in patients with chronic myeloid leukemia. Clin JOncol Nurs. 2011;15(6):660-667.

48. Kim TD, et al. Clinical cardiac safety profile of nilotinib. Haematologica. 2012;97(6):883-889.

49. Weigel MT, et al. Nilotinib in combination with carboplatin and paclitaxel is a candidate for ovarian cancer treatment. Oncology. 2014;87(4):232-245.

50. Tiwari AK, et al. Nilotinib potentiates anticancer drug sensitivity in murine ABCB1-, ABCG2-, and ABCC10-multidrug resistance xenograft models. Cancer Lett. 2013;328(2):307-317. 
51. Holbeck SL, et al. The National Cancer Institute ALMANAC: A comprehensive screening resource for the detection of anticancer drug pairs with enhanced therapeutic activity. Cancer Res. 2017;77(13):3564-3576.

52. Hidalgo M, et al. Patient-derived xenograft models: an emerging platform for translational cancer research. Cancer Discov. 2014;4(9):998-1013.

53. Hagenbuch B, Stieger B. The SLCO (former
SLC21) superfamily of transporters. Mol Aspects Med. 2013;34(2-3):396-412.

54. de Bruijn P, Moghaddam-Helmantel IM, Loos WJ, Mathijssen RH, Wiemer EA. The issue of non-specific binding of cabazitaxel. J Chromatogr B Analyt Technol Biomed Life Sci. 2013;932:74-75.

55. Meregalli C, et al. Bortezomib-induced painful neuropathy in rats: a behavioral, neurophysiological and pathological study in rats. Eur J Pain.
2010;14(4):343-350.

56. Filipski KK, Mathijssen RH, Mikkelsen TS, Schinkel AH, Sparreboom A. Contribution of organic cation transporter 2 (OCT2) to cisplatininduced nephrotoxicity. Clin Pharmacol Ther. 2009;86(4):396-402.

57. Goldman M, et al. The UCSC Cancer Genomics Browser: update 2015. Nucleic Acids Res. 2015;43(Database issue):D812-D817. 